\title{
Performance of the compensation comparison method for retinal straylight measurement: effect of patient's age on repeatability
}

\author{
A Cerviño, ${ }^{1}$ R Montes-Mico, ${ }^{1}$ S L Hosking ${ }^{2,3,4}$
}

${ }^{1}$ Optometry Research Group, Department of Optics, University of Valencia, Burjassot, Spain

${ }^{2}$ Department of Optometry and Visual Science, City University, London, UK; ${ }^{3}$ School of Life and Health Sciences, Aston

University, Birmingham, UK:

${ }^{4}$ Department of Ophthalmology, University of Melbourne,

Melbourne, Australia

Correspondence to:

Dr A Cerviño, Department of

Optics, University of Valencia, Dr

Moliner, 50, 46100 Burjassot,

Valencia, Spain; alejandro.

cervino@uv.es

Accepted 6 February 2008

Published Online First

17 April 2008

\section{ABSTRACT}

Aim: The assessment of repeatability and reproducibility of retinal straylight measurements with the C-Quant straylight meter (Oculus AG, Germany) and the effect of patient's age on the instrument performance are tested with a series of experiments.

Methods: First, 20 eyes from 20 subjects (mean age 26.9 (SD 2.7) years, mean refractive error -1.34 (2.72) D) were examined with the C-Quant straylightmeter, taking 10 consecutive readings. Five subjects were also examined on five consecutive days to assess reproducibility. Additionally, repeated measures of straylight from 84 subjects of ages ranging from 19 to 86 years (mean (SD): 42.4 (24.0) years) were retrospectively analysed to assess the effect of patient's age on repeatability.

Results: The results failed to show significant differences between the readings taken within the same session (mean (0.07), $p>0.05$ ) or between sessions (mean $(0.05), p>0.05)$. Variability of intrasession measurements was not significant for subjects of different age $(p=0.094)$.

Conclusion: It may be concluded that the C-Quant straylightmeter is repeatable and reliable for the assessment of retinal straylight in human eyes. Age of the patient does not decrease repeatability, even though they feel more insecure about their ability to perform the test.

Intraocular light scatter is light that has reflected, refracted, diffracted or experienced multiple combinations of all three from particles along the optical path of travel. ${ }^{1}$ There are five major sources that contribute to the total amount of ocular straylight: cornea, iris, sclera, retina and lens. ${ }^{2}$ It is assumed that for young healthy eyes, the total amount of straylight is $1 / 3$ by the cornea, $1 / 3$ by the lens and $1 / 3$ by the iris, sclera and retina. Obviously, these ratios change with age, pigmentation and specific pathologies. Corneal light scatter is constant with age ${ }^{34}$ and it may change with corneal defects or after corneal refractive surgery. ${ }^{5-7}$ The iris and the sclera scatter light depend on the patient's pigmentation ${ }^{8}$ (for example, brown eyes absorb more light and consequently produce less scatter than light eyes). Lens scatter increases with age, being greater in patients with cataracts. ${ }^{9}$ Finally, the retina produces light scatter to different locations being pigmentationdependent. $^{8}$

A clinical application of straylight measurement is to diagnose patients with complaints caused by a high degree of light scattering in the eyes such as lens opacities or corneal turbidity after laser corneal surgery. Considering that scatter light causes contrast loss in the final retinal image, the estimation of this parameter becomes very important in cataract and refractive surgery procedures. Several clinical devices have been developed to evaluate straylight and glare (ie, nyktotest, mesotest and the straylight meter). ${ }^{10} \mathrm{~A}$ recent computer version of the straylight meter has been designed to improve the clinical measurement of the ocular straylight. ${ }^{11}$

The C-Quant is a newly developed instrument to measure the retinal straylight using the "compensation comparison" method. In essence, this method presents exactly the same stimuli to the subject as the direct compensation method described in previous reports, ${ }^{12}$ and implemented in previous versions of the instrument. ${ }^{13}$ In contrast, in the compensation comparison method, two stimuli of the direct compensation method are presented to and compared by the subject simultaneously. ${ }^{11}$

The aim of the present study is to assess the effect that repeated measurement of retinal straylight has in the final values, which would be the optimum number of measurements needed to obtain an optimum reliability, and also the possible influence of the age of the patient on the repeatability of the measures is explored due to the psychometric character of the test.

\section{METHODS}

The present study involves three different experiments in order to determine first the variability of retinal straylight values with repeated measurements within one session, second the intersession variation and third if the subject's age has an impact on this repeatability. All the procedures followed the tenets of the Declaration of Helsinki and were approved by the Ethics Committee of the University of Valencia. Although both eyes could be used, since straylight is not symmetrical between eyes, only one eye was examined on each subject.

\section{Intrasession repeatability}

Twenty eyes from 20 young adults (mean age 26.9 (SD 2.7) years, mean refractive error -1.34 (2.72) D) were examined using the C-Quant stray-light meter (Oculus Optikgeräte $\mathrm{GmbH}$, Wetzlar, Germany). Ten consecutive measurements were taken on each eye without any compensation of refractive error. All measurements obtained were considered reliable by the system, that is, the estimated standard deviation (ESD) and quality factor for the psychometric sampling (Q) were 
Table 1 Mean logarithmic straylight value, $\log (\mathrm{s})$ and SD obtained from 10 measurements for each of the 20 patients within the same session

\begin{tabular}{lll}
\hline Patient & Mean log(s) & SD \\
\hline 1 & 0.91 & 0.12 \\
2 & 1.06 & 0.10 \\
3 & 0.69 & 0.06 \\
4 & 0.92 & 0.09 \\
5 & 0.62 & 0.07 \\
6 & 0.89 & 0.05 \\
7 & 0.72 & 0.05 \\
8 & 1.25 & 0.08 \\
9 & 0.80 & 0.06 \\
10 & 0.99 & 0.08 \\
11 & 0.99 & 0.09 \\
12 & 0.81 & 0.07 \\
13 & 1.05 & 0.07 \\
14 & 1.14 & 0.05 \\
15 & 0.91 & 0.05 \\
16 & 1.09 & 0.09 \\
17 & 1.00 & 0.04 \\
18 & 0.92 & 0.07 \\
19 & 1.05 & 0.13 \\
20 & 0.76 & 0.05 \\
\hline
\end{tabular}

lower than 0.08 and higher than 1.00 , respectively. The CQuant relies on these two parameters to identify whether the measurement can be considered reliable. The ESD, which the system identifies as reliable when lower than 0.08 , is based on a single assumed shape for the psychometric function, which depends itself on the critical modulation depth contrast and a parameter describing the lapse rate. Both parameters and the reasoning behind them have been described in detail elsewhere. ${ }^{21}$ A time interval of between $30 \mathrm{~s}$ and $1 \mathrm{~min}$ was allowed between measurements for the patient to rest from the task. Each single measurement lasted about 1.5-2 min.

\section{Intersession repeatability}

Additionally, five emmetropic subjects were measured three times on each of five sessions. These measurements were taken at the same time on five consecutive days in order to assess the intersession repeatability of the measurements.

\section{Effect of age of the patient on repeatability of measures}

In order to obtain this, the stray-light values of 84 subjects were retrospectively analysed. Subjects were between 19 and 86 years of age (mean (SD) 42.4 (24.0) years) with no ocular condition (mean spherical equivalent: $-1.12(1.09)$ D). Some of those subjects had previous cataract surgery, but since the aim of the present study is to determine the impact of age in the performance of the task involved in the psychometric determination of retinal straylight rather than the values themselves, they were included in the analysis.
Three consecutive measurements of retinal straylight considered as "reliable" by the instrument were obtained from each subject. The mean of those three was used for further statistical analysis. As suggested by the manufacturer, no correction was needed for the subjects examined to perform the compensation comparison test.

\section{Statistical analysis}

Normality of data distribution was assessed with the Kolmogorov-Smirnov test.

Repeated-measures analysis of variance (ANOVA) was applied to determine the existence of significant differences between the measurements obtained within the same session and between sessions. ANOVA was used to determine the impact of patient's age in the variance of the repeated straylight measures obtained.

According to sample size calculations, for a critical $p$ value of 0.05 used to denote statistical significance, a minimum sample size of 20 subjects would be sufficient to detect statistical significance for a change in light scattering of 0.10 log units among sessions or between measurements taken within the same session, based on an anticipated mean difference in the change and an overall variability of 0.10 log units for an average of $1.00 \log$ units for the overall population and assuming a statistical power of 0.99 . To detect a difference of 0.05 in light scatter, using the same sample size the statistical power would be of 0.60 . This implies that sample size for the intersession repeatability is quite small for statistical power but sufficient to offer a valid estimation of intersession variance through the intersession SD, also when checked against intrasession SD values.

\section{RESULTS}

\section{Intrasession repeatability}

Table 1 shows the mean and SD of the straylight parameter, in log units, obtained for the sample in the 10 consecutive measurements. Analysis of variance (ANOVA) did not show any statistically significant differences between the measurements taken within the same session. Figure 1 however shows that SD increases with the number of measurements, possibly due to tiredness of the subject examined due to the subjective character of the test and its duration (around $2 \mathrm{~min}$ ); it also shows little variation in SD throughout the 10 consecutive readings. The mean SD was 0.07 log units.

\section{Intersession repeatability}

Table 2 shows the straylight values and SD throughout the five sessions. Analysis of variance (ANOVA) did not show any statistically significant differences between the measurements obtained in the five sessions, although some degree of variability up to $0.22 \log$ units can be seen in fig 2 , which could possibly be attributed to normal individual variations. The mean intersession $\mathrm{SD}$ was 0.05 .

Table 2 Mean logarithm of the stray light value, $\log (\mathrm{s})$ and SD from the three measurements obtained on the five sessions taken on consecutive days

\begin{tabular}{|c|c|c|c|c|c|c|c|c|c|c|c|}
\hline \multirow[b]{2}{*}{ Patient } & \multirow[b]{2}{*}{ Intersession SD } & \multicolumn{2}{|c|}{ Session 1} & \multicolumn{2}{|c|}{ Session 2} & \multicolumn{2}{|c|}{ Session 3} & \multicolumn{2}{|c|}{ Session 4} & \multicolumn{2}{|c|}{ Session 5} \\
\hline & & $\log (s)$ & SD & $\log (\mathbf{s})$ & SD & $\log (s)$ & SD & $\log (s)$ & SD & $\log (s)$ & SD \\
\hline 1 & 0.03 & 0.84 & 0.05 & 0.86 & 0.04 & 0.81 & 0.02 & 0.85 & 0.04 & 0.90 & 0.08 \\
\hline 3 & 0.08 & 0.76 & 0.02 & 0.78 & 0.02 & 0.81 & 0.05 & 0.81 & 0.04 & 0.95 & 0.03 \\
\hline 4 & 0.09 & 0.90 & 0.03 & 0.94 & 0.04 & 0.95 & 0.04 & 1.12 & 0.03 & 1.04 & 0.04 \\
\hline 5 & 0.05 & 0.88 & 0.03 & 0.84 & 0.02 & 0.77 & 0.03 & 0.90 & 0.04 & 0.86 & 0.03 \\
\hline
\end{tabular}




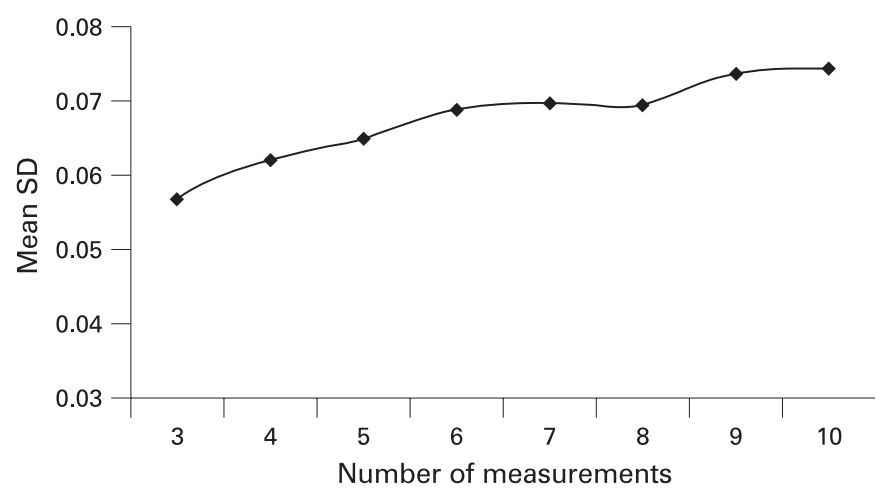

Figure 1 Variation of the mean SD from the 20 eyes with the number of measurements taken.

\section{Effect of age of patient on repeatability}

ANOVA showed, as expected, a significant increment of retinal straylight values with increasing age $(p<0.001)$ (fig 3$)$. Repeatability was not significantly different for patients of different age ( $p=0.094)$ (fig 4$)$.

\section{DISCUSSION}

Direct compensation method for the determination of retinal straylight has been successfully used in a number of studies and has been widely reported in literature as a useful approach in the study of optical performance in cataract, ${ }^{214}$ corneal dystrophies $^{15}$ or retinal disease, ${ }^{16-18}$ as well as the effects of ocular lubricants, ${ }^{19}$ laser refractive therapy ${ }^{520}$ or radial keratotomy. ${ }^{7}$ Recently, a modification to the original method has been made in order to make it more suitable for clinical use in a large scale. This new method was named the compensation comparison method, and has also been described in detail in the literature, ${ }^{11}$ resulting in a reliable instrument for the measurement of retinal straylight in clinical settings and successfully used in large-scale studies (see GLARE study: http://www.glare.be). However, all the studies reported to the authors' knowledge have been performed taking no more than two repeated measurements on each of the subjects. The aim of the present study was to assess the effect that repeated measurement of retinal straylight had in the final values, and which would be the optimum number of measurements needed to obtain an optimum reliability.

When repeated measures are performed using a test in which a bright light flickers at different intensity levels, and we require a response from the patient, we would expect some kind of effect of multiple consecutive readings, that is, the last readings would be less reliable than the first readings due to the sustained psychometric effort that would make the task more difficult to perform. In a previous study describing and assessing the procedure used here, Franssen et $a l^{11}$ found an overall SD of repeated measures between 0.06 and $0.1 \mathrm{log}$ units, which agrees with our findings of SD between 0.04 and $0.13 \log$ units. This high repeatability suggests that the system is reliable and useful for detecting clinically significant stray light values.

The C-Quant relies on two parameters to identify whether the measurement can be considered reliable or another measurement should be taken. One of these parameters is the ESD, which the system identifies as reliable when lower than 0.08 and is based on a single assumed shape factor for the psychometric function, which itself depends on the critical

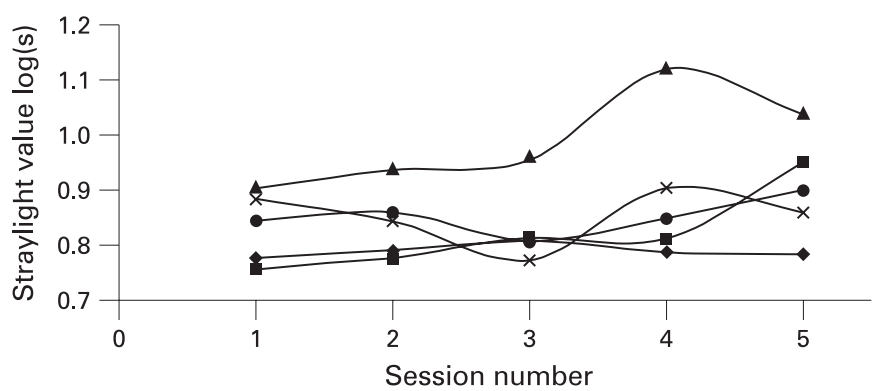

Figure 2 Plot of the variation of the mean straylight value $[\log (\mathrm{s})]$ obtained for the five subjects throughout the five sessions taken on five consecutive days. Error bars were omitted for clarity, but were lower than 0.1 in all cases, as can be interpreted from table 2 .

modulation depth contrast and a parameter describing the lapse rate. Both parameters and the reasoning behind them have been described in detail elsewhere. ${ }^{21}$ In a recent publication assessing the reliability of the method, Coppens et al showed ESD to be efficient for detecting unreliable measurements. ${ }^{21}$ From the results presented here, it can be seen that measurements considered reliable by the instrument are very repeatable both intra- and intersession.

Another issue that might affect the readings would be the ability of patients to perform the test. With increasing age, patients tend to feel more insecure about their ability to perform tests that require a direct response from them. During clinical determination of retinal straylight, it was noticed that many patients of considerable age would feel very worried during testing because they felt they did it wrong, were too slow or got confused about pressing the right button. One of the aims of the present study arose as a result of these observations. The results reported here show that the compensation comparison method is not affected by this, as long as the measurement obtained is considered as "reliable" by the instrument using the two parameters previously described, ESD and $\mathrm{Q}$.

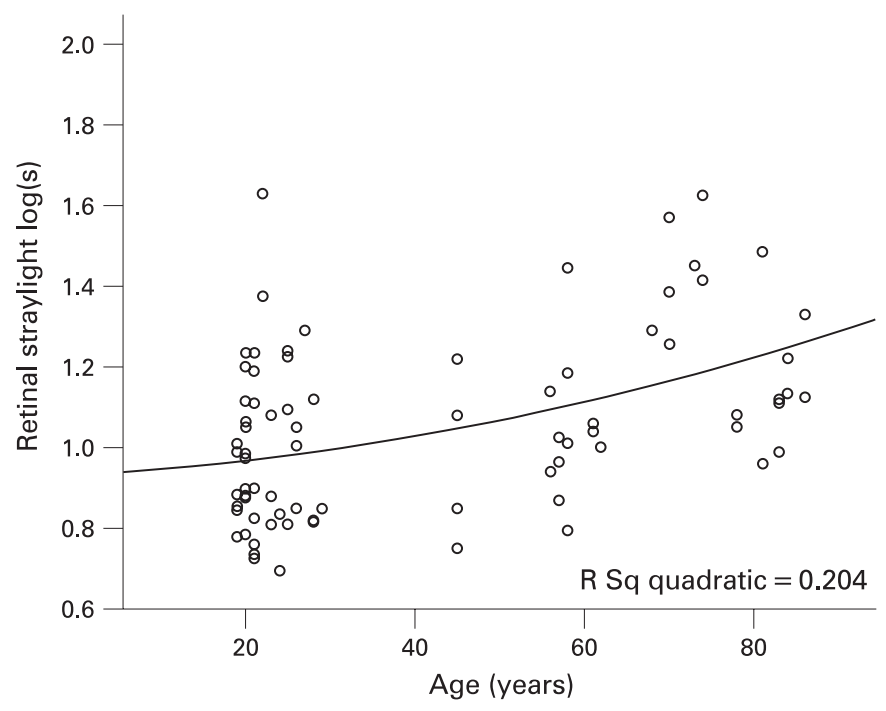

Figure 3 Scatter plot showing the retinal straylight values obtained in the sample analysed as a function of the age of the patient. The sample included clinical patients. 


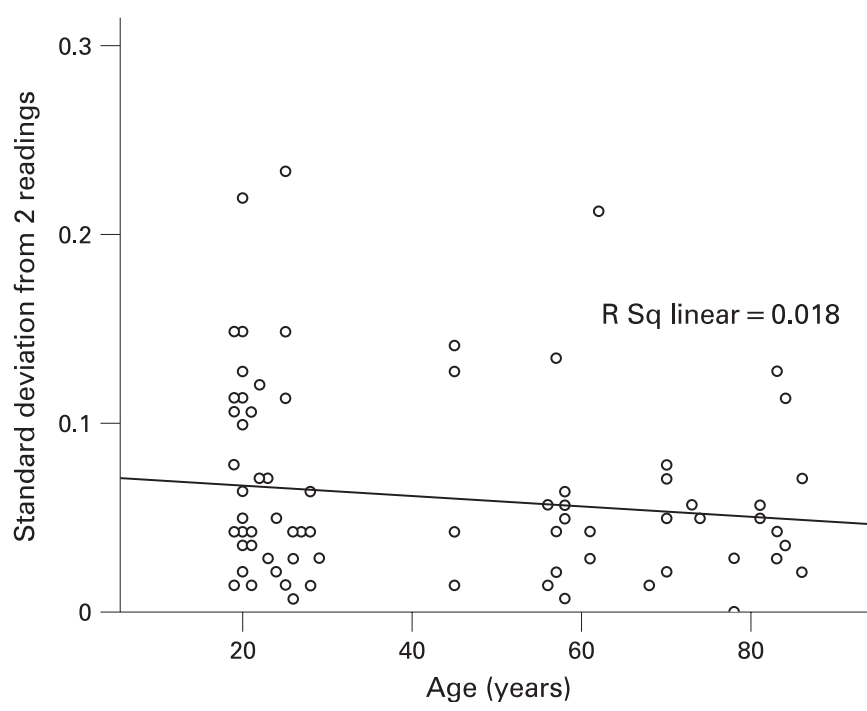

Figure 4 Scatter plot showing the standard deviation obtained from three consecutive readings on each patient as a function of the age of the patient.

In summary, the C-Quant stray-light meter is a very intuitive, easy-to-use clinical tool that provides reliable, repeatable measurements of retinal straylight in human eyes irrespective of age. Contrary to possible initial impressions, more measurements do not increase the reliability of the values obtained, and a balance between number of measurements and time required for the patient to perform the test is crucial. From the results obtained in the present study, three valid consecutive measurements are sufficient to obtain a reliable value for retinal straylight (although two measurements would suffice). Furthermore, the repeatability of the measurements is not influenced by the age of the patient.

Competing interests: None.

\section{REFERENCES}

1. Bohren C. Scattering by particles. In: Optical Society of America, ed. Handbook of optics. 2nd ed. New York: McGraw-Hill, 1995.

2. de Waard PW, JK IJ, van den Berg TJ, et al. Intraocular light scattering in agerelated cataracts. Invest Ophthalmol Vis Sci 1992;33:618-25.

3. Ijspeert J, de Waard PW, van den Berg TJ, et al. The intraocular straylight function in 129 healthy volunteers; dependence on angle, age and pigmentation. Vision Res 1990;30:699-707.

4. van den Berg TJ. Analysis of intraocular straylight, especially in relation to age. Optom Vis Sci 1995; 12:52-9.

5. Veraart HG, van den Berg TJ, Hennekes R, et al. Stray light in photorefractive keratectomy for myopia. Bull Soc Belge Ophtalmol 1993;249:57-62.

6. Veraart HG, van den Berg TJ, Hennekes R, et al. Stray light in photorefractive keratectomy for myopia. Doc Ophthalmol 1995;90:35-42.

7. Veraart HG, van den Berg TJ, JK IJ, et al. Stray light in radial keratotomy and the influence of pupil size and straylight angle. Am J Ophthalmol 1992;114:424-8.

8. van den Berg TJ, JK IJ, de Waard PW. Dependence of intraocular straylight on pigmentation and light transmission through the ocular wall. Vision Res 1991;31:1361-7.

9. de Wit G, Franssen L, Coppens JE, et al. Simulating the straylight effects of cataracts. J Cataract Refract Surg 2006:32:294-300.

10. van Rijn LJ, Nischler C, Gamer D, et al. Measurement of stray light and glare: comparison of Nyktotest, Mesotest, stray light meter, and computer implemented stray light meter. Br J Ophthalmol 2005;89:345-51.

11. Franssen L, Coppens JE, van den Berg TJ. Compensation comparison method for assessment of retinal straylight. Invest Ophthalmol Vis Sci 2006;47:768-76.

12. van den Berg TJ. Importance of pathological intraocular light scatter for visual disability. Doc Ophthalmol 1986;61:327-33.

13. van den Berg TJTP, IJspeert, J. K. Clinical assessment of intraocular straylight. Appl Opt 1992;31:3694-6.

14. Meacock WR, Spalton DJ, Boyce J, et al. The effect of posterior capsule opacification on visual function. Invest Ophthalmol Vis Sci 2003;44:4665-9.

15. van den Berg TJ, Hwan BS, Delleman JW. The intraocular straylight function in some hereditary corneal dystrophies. Doc Ophthalmol 1993:85:13-9.

16. Alexander KR, Fishman GA, Derlacki DJ. Intraocular light scatter in patients with retinitis pigmentosa. Vision Res 1996;36:3703-9.

17. Grover S, Alexander KR, Choi DM, et al. Intraocular light scatter in patients with choroideremia. Ophthalmology 1998;105:1641-5.

18. Grover S, Alexander KR, Fishman GA, et al. Comparison of intraocular light scatter in carriers of choroideremia and X-linked retinitis pigmentosa. Ophthalmology 2002;109:159-63.

19. Veraart HG, van den Berg TJ. Ocular lubricants and intraocular stray light. Doc Ophthalmol 1992;82:29-31.

20. Harrison JM, Tennant TB, Gwin MC, et al. Forward light scatter at one month after photorefractive keratectomy. J Refract Surg 1995;11:83-8.

21. Coppens JE, Franssen L, van Rijn LJ, et al. Reliability of the compensation comparison stray-light measurement method. J Biomed Opt 2006;11:34027. 